\title{
Prediction of outcome in adults with severe falciparum malaria: a
} new scoring system

\author{
Saroj K Mishra*1, Pinaki Panigrahi², Rajalaxmi Mishra ${ }^{3}$ and Sanjib Mohanty ${ }^{1}$
}

Address: ${ }^{1}$ Department of Internal Medicine, Ispat General Hospital, F-139, Sector-19, Rourkela, 769 005, Orissa, India, ${ }^{2}$ Department of Pediatrics, Epidemiology and Preventive Medicine, University of Maryland School of Medicine, Baltimore, MD, USA and ${ }^{3}$ Department of Mathematics, Sushilavati Government Women's College, Rourkela, Orissa, India

Email: Saroj K Mishra* - sarojrkl@gmail.com; Pinaki Panigrahi - ppanigrahi@pol.net; Rajalaxmi Mishra - mamimath@rediffmail.com; Sanjib Mohanty - ighmalaria@yahoo.com

* Corresponding author

Published: 27 February 2007

Malaria Journal 2007, 6:24 doi:10.1186/1475-2875-6-24

This article is available from: http://www.malariajournal.com/content/6/l/24

(C) 2007 Mishra et al; licensee BioMed Central Ltd.

This is an Open Access article distributed under the terms of the Creative Commons Attribution License (http://creativecommons.org/licenses/by/2.0), which permits unrestricted use, distribution, and reproduction in any medium, provided the original work is properly cited.
Received: 6 August 2006

Accepted: 27 February 2007

\begin{abstract}
Background: Mortality of falciparum malaria is related to the presence of severe complications. However, no scoring system is available to predict outcome of these patients. The aim of this paper was to devise a simple and reliable malaria prognosis score (MPS) to predict the outcome of adults with severe malaria.

Methods: All slide-positive severe falciparum malaria patients admitted to Ispat General Hospital were studied. Eight clinical parameters that may potentially differentiate or influence the outcome were identified to predict recovery or death

Results: Of 248 severe malaria cases, 35 died. There were 212 adults ( 34 deaths) and 36 children (one death). The malaria score for adults was $(M S A)=I$ (severe anaemia) +2 (acute renal failure) +3 (Respiratory distress) +4 (cerebral malaria). The MSA ranges from 0 to 10. The mortality was $2 \%$ for MSA 0-2; $10 \%$ for MSA 3-4, 40\% for MSA 5-6 and $90 \%$ for MSA 7 or more. The sensitivity is $89.9 \%$ and positive predictive value is $94.1 \%$ when 5 is taken as the cut off value.

Conclusion: MSA is a simple and sensitive predictor. It can be administered rapidly and repeatedly to prognosticate the outcome of severe malaria in adults. It can help the treating doctor to assess the patient as well as to communicate to the relatives of the patients about prognosis. The score needs revalidation in other geographical areas.
\end{abstract}

\section{Background}

Falciparum malaria is a leading cause of mortality and morbidity in developing countries [1]. Severe falciparum malariais associated with various complications. The WHO enumerates a list of complications [1], but the importance of each complication has not been assigned. It is well known that the presence of multiple complications results in increased mortality. The presence of pregnancy makes the outcome disastrous [2]. Similarly, Marsh et al reported respiratory distress as an indicator of lifethreatening malaria in African children [3,4]. Molyneux et al described the clinical and prognostic indicators of cerebral malaria in children [5]. However, no prediction scoring system is yet available to prognosticate the outcome in severe malaria among adult patients.

A simple scoring system is described to predict mortality in any severe and complicated malaria, based on available 
variables at the bedside with minimum laboratory parameters. The Malaria Score for adults (MSA) can be utilized at any time, and not as a "once only" predictor.

\section{Methods}

\section{Study area}

The study was carried out at Ispat General Hospital, Rourkela, in the district of Sundergarh, in the northwestern part of State of Orissa, India. It is a 700 bed hospital, which caters to the medical needs of more than 500,000 populations. Being a large and well-equipped hospital, over the years it has established as a tertiary care center for seriously ill patients from the adjoining districts of Orissa, Jharkhand, Chhatisgarh, Madhya Pradesh and Bihar.

\section{Patients}

This hospital-based study was conducted on patients over 12 years of age presenting with falciparum malaria who met the WHO criteria of severe and complicated falciparum malaria [1]. It includes cerebral malaria (unrousable coma), severe anaemia (haemoglobin $<5 \mathrm{~g} / \mathrm{dL}$ ), renal failure (serum creatinine $>3 \mathrm{mg} / \mathrm{dl}$ ), adult respiratory distress syndrome, hypoglycaemia (glucose $<40 \mathrm{mg} / \mathrm{dl}$ ), circulatory collapse or shock, disseminated intravascular coagulation, repeated generalized convulsions, acidosis ( $\mathrm{pH}<7.25)$, macroscopic haemoglobinuria, hyperparasitemia ( $>5 \%$ ) or jaundice (bilirubin $>3 \mathrm{mg} / \mathrm{dL}$ ). All the patients were symptomatic.

A detailed clinical evaluation was made with particular attention to neurological status, respiratory, renal condition and other complications. A sample of $5 \mathrm{ml}$ heparinized venous blood was collected for determination of plasma glucose, urea, serum creatinine, bicarbonate, serum electrolytes, bilirubin, alanine aminotransferase, hemoglobin, and for a total and differential leukocyte count and a parasite count. Soon after confirmation of the presence of asexual forms of Plasmodium falciparum in the peripheral blood smear, injection quinine dihydrochloride $10 \mathrm{mg} / \mathrm{Kg}$ body weight in $5 \%$ dextrose or in dextrose saline was infused over a period of 4 hours, every 8 hours, until the patient was able to take the drug orally. Oral quinine sulphate was given to complete the total duration of seven days therapy. Supportive measures like maintenance of hydration, antibiotics for any concurrent infections, blood transfusion, dialysis, ventilator support etc. were given according to individual needs. The patients who recovered and those who expired were grouped and the statistical analyses were performed [6].

\section{Statistical analysis}

The clinical parameters associated with survival and deaths were compared by using) Chi-square $\left(\chi^{2}\right)$ test with Fisher exact test where applicable; Odds ratio (OR) with
95\% Confidence Interval. Those parameters which were significant were used for the multivariate analysis to get a linear discriminant equation showing the relationship between malaria score and the variables, i.e., a formula in the form of Malaria Prediction Score (MPS) $=a+b_{1} x_{1}+$ $\mathrm{b}_{2} \mathrm{x}_{2}+\mathrm{b}_{3} \mathrm{x}_{3}+\ldots+\mathrm{b}_{\mathrm{n}} \mathrm{x}_{\mathrm{n}}$

where $\mathbf{a}=$ constant, $\mathbf{b}_{\mathbf{i}}=$ discriminant coefficients $(\mathrm{i}$ $=, 2,3 \ldots \ldots \ldots \ldots)$ and $x_{i}=$ independent variables $(i=1,2,3 \ldots$ (... n).

\section{Results}

The study to develop the score included 248 severe malaria patients with 35 deaths. There were 36 children (one death) and 212 adults (34 deaths). All the variables were analysed (viz., age, gender, presence of cerebral malaria, seizures, hyperpyrexia, hypotension, severeanaemia, haemolysis, acute renal failure, jaundice, hypoglycaemia, respiratory complications needing ventilator support and pregnancy. Of these, cerebral malaria, severe anaemia, acute renal failure, and need of ventilator were identified as indicators of high risk. The adults were more vulnerable than the children. Presence of cerebral malaria, severe anaemia, renal failure or respiratory distress needing ventilatory support were highly significant whereas adult age or pregnancy were significant to a lesser degree. There was no difference in the mortality between males and females. The presence of haemolysis, hypoglycaemia or hypotension were not significantly different in the two groups. After multiple regression analysis, the results were studied to identify as few variables as possible to predict the outcome:

The formula for Malaria prediction Score (MPS) thus evolved was as follows:

MPS $=2.13+0.02 \times($ Age $)+0.25 \times($ Creat $)-0.24 \times(\mathrm{Hb})$

+ 3.05 (Cerebral) +0.8 (Pregnancy $)+0.8$ (Ventilator $)$

(where, Age = age in years; Creatinine is in $\mathrm{mg} / \mathrm{dl}$, Haemoglobin in G/dl; Presence of Pregnancy, cerebral malaria or ventilatory support, when present $=1$, when absent $=0$.)

However, there were some practical problems in MPS : a) only one death from 36 children and b) the laboratory data for serum creatinine, bilirubin, haemoglobin and blood sugar must be available to get the MPS. This would delay in calculating the MPS, and thus it cannot be administered at the time of evaluation. Hence, the data from those patients aged above 12 years were analysed to develop the Malaria score to predict the outcome among adult patients with severe malaria. The clinical parameters of those who survived vs those who expired are given in Table 1. From the table it signifies the importance of four 
variables. Viz., cerebral malaria, acute renal failure, severe anaemia and repiratory failure requiring ventilator. The presence of hypoglycemia, jaundice, seizures or pregnancy did not adversely influence the survival.

So the score was accordingly modified (a) to include only the adults, and (b) the presence or absence of a clinical parameter was denoted as 1 or 0 . Thus the malaria score for adults was $(M S A)=1($ severe anaemia $)+2$ (acute renal failure) + 3(Respiratory distress) + 4 (cerebral malaria) The MSA ranges from 0 to 10 . The mortality was $2 \%$ for MSA $0-2 ; 10 \%$ for MSA $3-4,40 \%$ for MSA 5-6 and $90 \%$ for MSA 7 or more. The sensitivity is $89.9 \%$, specificity $70.6 \%$, and positive predictive value is $94.1 \%$ when 5 is taken as the cut off value.

\section{Discussion}

The clinical course of severe malaria is variable depending on the presence of one or several complications. The fatality rate of cerebral malaria, renal complications, acute lung injury is very high and they cannot be grouped together as similar to hypoglycaemia, hypotension, hyperpyrexia or seizure. It iscrucial to prognosticate the cases accordingly. As it was observed, the presence of haemolysis, hyperpyrexia, hypoglycaemia or hypotension alone was not a major factor; and not a single patient died due to any one of these. Moreover, combination if these also did not end with fatality. The prognostic indicators as suggested by Molyneux et al [5] for childhood cerebral malaria includes four neurological parameters (witnessed seizures, coma score of 0 , signs of decerebration, absent corneal reflexes); and three laboratory parameters (blood glucose level below $2.2 \mathrm{mmol} / \mathrm{l}$, parasitaemia $>10^{6} \mathrm{ring}$ forms, total leukocyte count $>15 \times 10^{9} / 1$ ). This index is applicable only for the cerebral malaria cases, and there is no indicator for other forms of severe malaria. Moreover, each of the parameter was given a uniform weightage of 1 . The positive predictive value of 4 or more of the above for an unsatisfactory outcome was $83 \%$ but sensitivity was only $66 \%$.

Lewallen et al described the retinal findings to predict the outcome in cerebral malaria [7]. Papilloedema and extramacular retinal oedema are prognostically significant. The relative risk of death in patients with papilloedema was 6.7 times more than the patients without.

Marsh et al stressed the importance of respiratory complications as a simple bedside prognostic indicator in children with cerebral malaria [3]. This could identify $84.4 \%$ fatal cases. In an editorial in the same issue it was commented that one cannot exclude the possibility of other respiratory illnesses and incidental $P$. falciparum parasitaemia. The definition of different degrees of severity of respiratory distress needs refinement. Many of the components of the clinical picture are subjective thus increasing the risk of inter observer variation [4]. In the present study, respiratory distress needing ventilatory support is considered which thus eliminated the observer bias. In most of the studies the attention is concentrated on cerebral malaria. Here all complications were analyzed and their contributing factors were taken into account. All the parameters are unambiguous and free from subjective error.

Presence of multiple complications made the management difficult. Even in the tertiary care centers the management becomes challenging as a number of complications can appear at the time of admission or even during the course of hospitalization. To predict the survival of the index case is therefore very difficult and demanding from the relatives of the patient. A guarded opinion is invariably put forth, depending on the personal experience, intuition, clinical acumen of the physician; as no predictive scoring system yet available. The literature is scant about the relative risk of the complications in malaria In this situation, the severity classification APACHE-II [8] may be used, but it is too exhaustive and cumbersome. So the need was felt to develop malaria prediction score for adults, which can be used even in a small town hospital, to decide the continuation of treatment or referral to a higher center.

In some clinical situations, scoring methods are available for prediction of fatality (viz. burns [9], acute pancreatitis [10], stroke [11] etc); where the aim is prediction of mor-

Table I: Comparison of different parameters among those who survived \& those who died.

\begin{tabular}{|c|c|c|c|c|}
\hline Parameters* & Expired $(n=34)$ & Recovered $(n=178)$ & OR $(95 \% \mathrm{Cl})$ & $\mathrm{P} * *<$ \\
\hline Cerebral malaria & 32 & 89 & $16.0(3.85-140)$ & 0.000 \\
\hline Seizures & 02 & 15 & $0.68(0.07-3.16)$ & $0.465^{a}$ \\
\hline Severe anaemia & 12 & 27 & $3.05(1.25-7.4)$ & 0.006 \\
\hline Jaundice & 12 & 88 & $0.56(0.24-1.27)$ & 0.131 \\
\hline Acute renal failure & 13 & 22 & $4.39(1.78-10.8)$ & 0.001 \\
\hline Hypoglycaemia & 01 & 04 & $1.32(0.03-13.86)$ & $0.807^{a}$ \\
\hline Ventilatory support & 08 & 05 & $10.56(2.77-43.88)$ & $0.000^{\mathrm{a}}$ \\
\hline Pregnancy & 06 & 19 & $1.79(0.54-5.20)^{\prime}$ & $0.249 a$ \\
\hline
\end{tabular}

* Described in the text $* * \chi^{2}$ test a: by Fisher exact test 
tality and hospital stay. In the case of malaria, if one can predict the survival, it is usually a full cure with rare sequel. So it is very important to predict the case survival. A scoring system though statistically significant has ultimately limited clinical use if (i) it is too cumbersome and difficult to remember, (ii) it has too many subjective variables or (iii) it requires sophisticated variables. Thus, a simple scoring system is always sought, so that only simple calculations would be needed, making it readily applicable at the bedside. The laboratory tests are routine and readily available; the clinical parameters are unambiguous without any subjective variation. The sensitivity of MSA is $89.9 \%$ and positive predictive value is $94.1 \%$. It predicts better than the Malawian prognostic index (for cerebral malaria) $83 \%$ sensitivity of $66 \%$. It is hoped that this scoring system can be validated in different geographical areas and subsequently be utilized at several places.

\section{Authors' contributions}

All the authors conceptualized the study, participated significantly in the analysis, drafting of the manuscript and writing the final version of the paper. SKM and RM contributed towards the mathematical modeling.

\section{Acknowledgements}

We express our sincere thanks to Dr DN Mohapatra for his encouragement and to Dr BS Das, Dr JK Pattnaik and Dr SK Satpathy for their constructive criticism.

\section{References}

I. WHO: Severe and complicated malaria. Trans $R$ Soc Trop Med Hyg 1990, 84(suppl 2): I-65.

2. Mishra SK, Mohanty S, Satpathy SK, Pattnaik JK: Complicated falciparum malaria during pregnancy. Jr Obstet Gynec India 1998, 48:31-34.

3. Marsh K, Forster D, Waruiru C, et al.: Indicators of life threatening malaria in African children. $N$ Engl J Med 1995, 332: |399-| 404.

4. Taylor TE, Molyneux ME: Something new out of Africa (editorial). $N$ Engl J Med 1995, 332:|44|-43.

5. Molyneux ME, Taylor TE, Wirima JJ, Borgstein A: Clinical features and prognostic indicators in paediatric cerebral malaria: a study of I3I comatose Malawian children. QJM I989, 7I:44I-459.

6. Machin D, Gardener MJ: Calculating the confidence intervals for survival data analysis. BMJ I989, 296:I369-I37I.

7. Lewallen S, Bakkar H, Taylor TE, Wills BA, Courtright P, Molyneux ME: Retinal findings: prediction of outcome in cerebral malaria. Trans Roy Soc Trop Med Hyg 1996, 90: I44-I46.

8. Knauss WA, Drapper EA, Wagner DP, Zimmerman JE: APACHE II : a severity of disease classification system. Crit Care Med 1985, | 3:818-29.

9. Ryan CM, Scenfields DA, Throppe WP, Schender RL, Carsen EH, Tompkins RG: Objective assessment of the probability of death from burn injuries. N Engl J Med 1998, 338:362-66.

10. Wilson C, Heath DI, Imrie CW: Prediction of outcome in acute pancreatitis: a comparative study of APACHE-II, clinical assessment and multiple factor scoring system. Br J Surg 1990, 77:1260-64.

II. Poungvarin N, Viriyavejakul A, Komontri C: Siriraj stroke score and validation study to distinguish between ICH from infarction. $B M]$ I991, 302:1565-67.
Publish with Biomed Central and every scientist can read your work free of charge

"BioMed Central will be the most significant development for disseminating the results of biomedical research in our lifetime. "

Sir Paul Nurse, Cancer Research UK

Your research papers will be:

- available free of charge to the entire biomedical community

- peer reviewed and published immediately upon acceptance

- cited in PubMed and archived on PubMed Central

- yours - you keep the copyright

Submit your manuscript here:

http://www.biomedcentral.com/info/publishing_adv.asp
BioMedcentral 